\title{
Seminal fluid parameters of male partners of infertile couples seeking care at the Lagos University Teaching Hospital
}

\author{
Christian Chigozie Makwe', Aloy Okechukwu Ugwu ${ }^{1 *}$, Rufus Wale Ojewola ${ }^{2}$, \\ Chigozie Innocent Onyeze ${ }^{2}$
}

\begin{abstract}
${ }^{1}$ Department of Obstetrics and Gynecology, ${ }^{2}$ Department of Surgery, College of Medicine University of Lagos and Lagos University Teaching Hospital, Lagos
\end{abstract}

Received: 15 February 2021

Accepted: 12 March 2021

\section{*Correspondence:}

Dr. Aloy Okechukwu Ugwu,

E-mail: okeyugwu92@gmail.com

Copyright: (C) the author(s), publisher and licensee Medip Academy. This is an open-access article distributed under the terms of the Creative Commons Attribution Non-Commercial License, which permits unrestricted non-commercial use, distribution, and reproduction in any medium, provided the original work is properly cited.

\section{ABSTRACT}

Background: Seminal fluid analysis is essential in the evaluation of the male partner of infertile couple. Available data suggest an increase in male-related factors among infertile couples seeking fertility treatment. Seminal fluid analysis performed using standardized methods can identify several causes of male-factor infertility. The latest World Health Organization (2010) guidelines for the laboratory examination and processing of human semen allows for standardization and identification of the possible causes of male-factor infertility. This study aimed to determine the pattern of seminal fluid analysis of male partners of infertile couple presenting to the fertility (gynaecological and urological) clinics of the Lagos University Teaching Hospital (LUTH).

Methods: This was a retrospective study of the semen parameters of male partners of infertile couple that were evaluated at LUTH, from January 2018 to December 2019. The medical records of all male partners of infertile couples who had seminal fluid analysis were reviewed and the relevant seminal fluid parameters were collected using a standardized questionnaire.

Results: A total of 604 male partners of couples with infertility sought fertility treatment during the study period. Out of the $604,502(83.1 \%)$ of the participants had complete seminal fluid result and these data were included in final analysis. The mean (SD) age of subjects was 42.1 (7.7) years. About three-quarter (73.1\%) of the subjects have abnormal semen parameters. The most frequent semen abnormality was asthenozoospermia (55.8\%) followed by oligozoospermia (39.4\%).

Conclusions: Male factor is still common cause of infertility among infertile couple seeking treatment in our environment.

Keywords: Male infertility, Semen analysis, Infertility, Oligozoospermia, Asthenozoospermia, Nigeria

\section{INTRODUCTION}

Infertility is a common public health problem in the tropics with important psychologic, economic, social, and medical implications because procreation is believed to have greater cultural and social values. ${ }^{1,2}$ It has remained a very sensitive issue as involuntary childlessness is a source of social stigma, marital disharmony and domestic violence; especially to the female partner. ${ }^{3}$ This arises from societal misconceptions which insinuates that failure to get pregnant connotes a female problem and the fact that some male partners equate adequate erection to fertility and therefore becomes elusive to investigations and treatment. ${ }^{1,3}$

Infertility affects about $15-20 \%$ of couples and refers to the inability of a couple to achieve and sustain pregnancy 
after at least one year of regular unprotected penetrative and ejaculatory vaginal intercourse with an adult of opposite sex. ${ }^{1,3}$ The causes could be attributed to either the female or male partner or both. The causes of male factor infertility may be classified as pre-testicular, testicular and post testicular factors. ${ }^{1-5}$ These factors usually result from abnormalities in the semen quantity or quality, hence, seminal fluid analysis for deficiencies in semen quality and quantity may be used as a surrogate measure of male fecundity. ${ }^{2,5,6}$ This analysis includes semen volume and $\mathrm{pH}$, microscopy for sperm concentration, count, motility, morphology, vitality, debris and agglutination and leucocyte count. ${ }^{7}$

Seminal fluid analysis is generally inexpensive laboratory test, and the services are readily available in most fertility clinics. ${ }^{2,5}$ It is therefore imperative to identify the causes of these abnormal seminal fluid parameters and to offer solutions early. ${ }^{8-11}$

This study therefore seeks to determine pattern of seminal fluid analysis of male partners of infertile couple, seeking fertility treatment at the gynaecology and urology clinics of the Lagos University Teaching Hospital (LUTH).

\section{METHODS}

This study was carried out at the gynaecology and urology outpatients' clinics of LUTH, a tertiary hospital. The Lagos University Teaching Hospital (LUTH) is the teaching hospital of the College of Medicine, University of Lagos. The hospital offers specialized treatment to infertile couple seeking care; including in-vitro fertilization. It acts mainly as a referral center for other government-owned and private hospitals in Lagos state. Gynaecology clinic holds Monday through Thursday while the urology clinic holds on Mondays and Thursdays.

This was a retrospective study of cases of infertility that were evaluated at LUTH over a two-year period, between January 2018 and December 2019. All the medical records of infertile couples with semen fluid analysis were reviewed and the relevant seminal fluid parameters (including semen volume, sperm concentration, motility, and morphology) were collected using a questionnaire.

Data were entered, cleaned and analyzed using the IBM Statistical Package for Social Sciences (SPSS Statistics) Version 23. Armonk, NY: IBM Corp. The categorical variables were summarized and presented as frequency distribution tables, while continuous variables were presented as mean (standard deviation).

\section{RESULTS}

A total of 604 male partners of couples with infertility sought fertility treatment during the study period. Out of the $604,502(83.1 \%)$ of the participants had complete seminal fluid result and these data were included in final analysis. Table 1 shows the age distribution of male partners of couples with infertility. The mean (SD) age of subjects was 42.1 (7.7) years with a range of 26 years to 76 years. About half of participants were aged between 35 and 44 years.

Table 1: Age distribution of male partners of infertile couples.

\begin{tabular}{|c|c|c|}
\hline & $\mathrm{N}=502$ n (\%) & Mean (SD) \\
\hline \multicolumn{3}{|c|}{ Age group (years) } \\
\hline $25-34$ & $83(16.3)$ & $42.1(7.7)$ \\
\hline $35-44$ & $249(49.6)$ & \\
\hline $45-54$ & $141(28.1)$ & \\
\hline$\geq 55$ & $29(5.8)$ & \\
\hline
\end{tabular}

Table 2: Seminal fluid parameter of male partners of infertile couples $\mathrm{N}=\mathbf{5 0 2}$.

\begin{tabular}{|c|c|c|}
\hline & $\mathbf{n}(\%)$ & Mean (SD) \\
\hline \multicolumn{3}{|c|}{ Semen Liquefaction Time (min) } \\
\hline$\leq 60$ & $483(96.2)$ & \\
\hline$>60$ & $19(3.8)$ & \\
\hline \multicolumn{3}{|c|}{ Semen Volume (mL) } \\
\hline $0.1-1.4$ & $74(14.7)$ & $2.9(1.5)$ \\
\hline $1.5-6.0$ & $415(82.7)$ & \\
\hline $6.0-9.0$ & $13(2.6)$ & \\
\hline \multicolumn{3}{|l|}{ PH } \\
\hline$<7.2$ & $29(5.8)$ & $7.6(0.3)$ \\
\hline$\geq 7.2$ & $473(94.2)$ & \\
\hline \multicolumn{3}{|c|}{ Sperm Concentration, $10^{6} / \mathrm{mL}$} \\
\hline 0.0 & $51(10.2)$ & $30.0(39.4)$ \\
\hline $0.01-14.9$ & $198(39.4)$ & \\
\hline$\geq 15$ & $253(50.4)$ & \\
\hline \multicolumn{3}{|c|}{ Total Sperm Number, $10^{6} /$ jaculate } \\
\hline$<39$ & $245(48.8)$ & $83.8(117.2)$ \\
\hline$\geq 39$ & $257(51.2)$ & \\
\hline \multicolumn{3}{|c|}{ Progressive Motility (\%) } \\
\hline$<32$ & $280(55.8)$ & $32.2(26.2)$ \\
\hline$\geq 32$ & $222(44.2)$ & \\
\hline \multicolumn{3}{|c|}{ Total Motility (\%) } \\
\hline$<40$ & $168(33.5)$ & $32.2(26.2)$ \\
\hline$\geq 40$ & $334(66.5)$ & \\
\hline \multicolumn{3}{|c|}{ Normal Sperm Morphology (\%) } \\
\hline$<4$ & $78(15.5)$ & $55.2(33.2)$ \\
\hline$\geq 4$ & $424(84.5)$ & \\
\hline \multicolumn{3}{|c|}{ White Blood Cell, $10^{6} / \mathrm{mL}$} \\
\hline$\leq 1$ & $372(74.1)$ & $1.5(3.2)$ \\
\hline$>1$ & $130(25.9)$ & \\
\hline
\end{tabular}

Table 2 shows the seminal fluid parameters of the study participants. Only $135(26.9 \%)$ of the participants had normal sperm parameters (volume, sperm concentration, progressive motility, and morphology) Table 3 shows the classification of sperm abnormalities. One-tenth of participants had azoospermia and two-fifth had 
oligozoospermia. The most frequent abnormality detected was asthenozoospermia in $280(55.8 \%)$ of participants.

Table 3: Classification of semen abnormalities.

\begin{tabular}{|ll|}
\hline & $\mathbf{N}=\mathbf{5 0 2} \mathbf{n}(\%)$ \\
\hline Oligoospermia & $51(10.2)$ \\
\hline Asthenozoospermia & $198(39.4)$ \\
\hline Teratozoospermia & $280(55.8)$ \\
\hline Oligoasthenozoospermia & $78(15.5)$ \\
\hline Oligotetratozoospermia & $129(25.7)$ \\
\hline Asthenoteratozoospermia & $19(3.7)$ \\
\hline OAT syndrome & $73(14.5)$ \\
\hline Hypospermia & $16(3.1)$ \\
\hline Hyperspermia & $74(14.7)$ \\
\hline
\end{tabular}

Table 4: Organisms isolated from seminal fluid culture.

\begin{tabular}{|ll|}
\hline Staphylococcus aureus & $\begin{array}{l}\text { N=70 } \\
\text { n }(\%)\end{array}$ \\
\hline Escherichia coli & $32(45.7)$ \\
\hline Candida albicans & $18(25.7)$ \\
\hline Pseudomonas aeruginosa & $8(11.4)$ \\
\hline Klebsiella spp & $5(7.1)$ \\
\hline Enterococcus faecalis & $4(5.7)$ \\
\hline
\end{tabular}

Of the 502 seminal fluid analysed, $432(86.1 \%)$ had no organism isolated while $70(13.9 \%)$ had organism isolated during culture. Table 4 shows the organisms isolated from seminal fluid culture. The most common organism isolated was Staphylococcus aureus in 32 $(45.7 \%)$ of semen with positive microbiological culture.

\section{DISCUSSION}

Infertility remains a challenge in this part of the world, and the cause may be linked to either or both partners. Semen analysis is a valid means of assessing male fertility. It was erroneously believed in some quarters, that the cause of infertility is essentially from the woman; however, this study has shown that about three-quarters of male partners have seminal fluid abnormalities which might be contributory to infertility in these couples.

In this study, about three-quarter $(73.1 \%)$ of the subjects had at least one abnormal semen parameter while some had a combination of abnormalities. This is similar to $66.7 \%$ reported in Delta state but higher than $30 \%$ reported in Ibadan and Abakaliki. ${ }^{1,5,7}$

The World Health Organization (WHO) in 2010 defined normal values for semen parameters as complete liquefaction within 60 minutes at room temperature, seminal volume of greater than or equal to $1.5 \mathrm{~mL}$, and a $\mathrm{pH}$ greater than or equal to 7.2. Other parameters comprise sperm concentration of greater than or equal to 15 million sperm cells per mL, total sperm count of 39 million per ejaculate, progressive motility of $32 \%$ or more, vitality of $58 \%$ or more live spermatozoa, total motility of $40 \%$ and a morphology of $4 \%$ or more of normal forms. ${ }^{6-8}$

The most common abnormality observed was asthenozoospermia, and only $44.2 \%$ of subjects had sperms with normal forward progressive motility, which is also similar to a study by Jeje et al that reported asthenozoospermia as the commonest semen abnormality. ${ }^{2}$ Abnormal sperm motility is a major factor contributing to infertility, as the sperm will be unable to ascend the female genital tract for fertilization. Male partners with asthenozoospermia may be offered assisted conception using intracytoplasmic sperm injection (ICSI). $9,12-14$

Other semen abnormalities noted in our study are azoospermia (11.4\%) and oligozoospermia (16.7\%). The frequency of azoospermia in this study is similar to $11.0 \%$ reported by Omo-Aghoja L et al in Niger delta region, but, slightly higher than $6.4 \%$ and $7.8 \%$ reported in Ibadan and Abakaliki, respectively., ${ }^{5,7}$ The proportion of subjects with oligozoospermia in this study is less than the $32.1 \%$ reported by Ajah, et al, and $22.8 \%$ reported by Omo-Aghoja L et al, but similar to that of Adeniji et al where it accounted for $18.9 \%$ of the abnormal semen parameters. Furthermore, teratozoospermia accounts for $0.8 \%$ of our seminal fluid abnormalities unlike previous studies by Adeniji and Ajah et al who reported $6.5 \%$ and $8.4 \%$ respectively. Some subjects had a combination of abnormalities with oligoasthenozoospermia accounting for $25.7 \%$, asthenoteratozoospermia (14.5\%), oligoteratozoospermia $\quad(3.7 \%) \quad$ and oligoasthenoteratozoospermia (OAT) syndrome $3.1 \%$. The oligoteratozoospermia of $3.7 \%$ observed in our study is similar to previous studies in Abakaliki and Lagos who reported $3.4 \%$ and $2.9 \%$, respectively; although differs in other parameters. ${ }^{7,14}$

Most of the patients in this study had normal liquefaction time, while almost half of the patients had sperm concentration less than normal. This is higher than findings by Adeniji et al, who reported one-quarter of patients in their study having semen abnormalities. ${ }^{1}$ This finding reaffirms the fact that male factor is a major contributor to infertility. This finding may also be as a result of the population of patients involved, as a community-based sampling may give a lower rate of abnormal semen analysis.

Most of our patients did not have any organism isolated from their semen culture. However, among those who had positive culture, staph aureus had the highest incidence. This is similar to findings by Emokpae et al, in his study involving 500 men. ${ }^{10}$ However, in developed countries, Chlamydia trachomatis, Ureaplasma urealyticum and enterobacteria are the most commonly 
isolated organisms. ${ }^{11}$ The high rate of staphylococcus infection may be linked with the method of collection as most patients used masturbation, although adequate hand and penile hygiene is usually advocated before semen collection. Bacterial infection is known to impair spermatogenesis, sperm function and may result in obstruction to the genital tract. ${ }^{11}$ However, the potential effects of bacterial infection may be reversible following prompt and adequate treatment with appropriate antibiotics. ${ }^{12}$ It is also not surprising that men in their fifth decade, who comprised majority of the patients, had the highest infection rate

Regarding volume of ejaculate, most patients produced between $1.5 \mathrm{ml}$ and $6 \mathrm{ml}$ which is considered normal. About $15 \%$ of patients had semen volume of less than $1.5 \mathrm{ml}$, and this may be a pointer to ejaculatory duct obstruction. Our study is also similar to other studies in Ibadan, Abakaliki, Ife and Lagos where no case of aspermia was found but different from that of a secondary health facility in Ebonyi with $12.3 \%$ males who had aspermia. ${ }^{1,7,13-15}$ This very rare abnormality is the complete absence of semen with ejaculation, due to either anejaculation or retrograde ejaculation. ${ }^{16}$ Aspermia should be differentiated from azoospermia which is lack of sperm cells in the semen.

\section{Limitations}

This is a retrospective study and suffers from the limitation of missing data which might affect the results. Furthermore, this is a facility-based study and may not reflect what happens in the general population.

\section{CONCLUSION}

Male factor is still common cause of infertility among infertile couple seeking treatment in our environment. Most of the male partners had obvious abnormality in their seminal fluid parameters, therefore, it is imperative to involve male partners in the evaluation of infertile couple.

\section{ACKNOWLEDGMENTS}

We thank the medical records staff for retrieving the case files for review. We also grateful to Antigha Esienanwan Cynthia for helping out with data entry.

\section{Funding: No funding sources}

Conflict of interest: None declared

Ethical approval: The study was approved by the Human Research Ethical Committee of the Lagos University Teaching Hospital. Informed consent was not required for this study as it depends on previously collected service data and a waiver was issued by the ethics committee

\section{REFERENCES}

1. Adeniji RA, Olayemi O, Okunlola MA, Aimakhu CO. Pattern of semen analysis of male partners of infertile couples at the University College Hospital, Ibadan. West Afr J Med. 2003;22(3):243-5.

2. Jeje EA, Alabia TO, Ojewola RW, Ogunjimi MA. Osunkoya SA. Male infertility: An Audit of 70 cases in a single centre. African Journal of Urol. 2016:22(3);223-6.

3. Umar AG, Abubakar AP, Mbakwe M, Ahmed Y, Garba JA, Nnadi DC. The Pattern of Seminal Fluid Analysis among Male Partners Attending an Infertility Clinic in a Nigerian Tertiary Health Institution. Open J Obstetr Gynecol. 2020:10;957-67.

4. Juneja P, Phukan PK, Changmai D. A study of abnormal semen parameters in infertile couples in Assam, India. Int J Reprod Contracept Obstet Gynecol. 2019;8(3):997-1000.

5. Ngwu M, Omo-Aghoja LO, Adeyinka AT. Pattern of Seminal Fluid Parameters and their Clinical Correlates Amongst Infertile Men in The Niger-Delta Region of Nigeria. Postgraduate Medic J Ghana. 2017:6(2);89-97.

6. World Health Organization Department of Reproductive Health and Research. World Health Organization Laboratory Manual for the Examination and Processing of Human Semen, 5th ed, World Health Organization, Geneva, Switzerland 2010.

7. Ajah LO, Ozumba BC, Onoh RC, Ezeonu PO, Iyoke CA, Ezeome IV, Seminal Fluid Features of Male Partners of Infertility Patients in Southeast Nigeria. African J Medic Health Sci. 2016:5(2);85-91.

8. Nasir AS, Agbo EB, Sani MY, Ali M. Assessment of microbiological quality of semen of male patients with infertility at Murtala Muhammad specialist hospital Kano, Nigeria. MOJ Bioequiv Availab. 2018;5(3):169-73

9. Nwofor AM, Ugezu AI. The difficulties encountered in the management of male infertility in Nnewi, Nigeria. Niger Med J. 2003;45(1):56-9.

10. Emokpae MA, Uadia PO, Sadiq NM. Contribution of bacterial infection to male infertility in Nigerians. Online Journal of Health and Allied Sciences. 2009;8(1).

11. Keck C, Gerber-Schäfer C, Clad A, Wilhelm C, Breckwoldt M. Seminal tract infections: impact on male fertility and treatment options. Human Reproduction Update. 1998;4(6):891-903.

12. Weidner W, Garbe C, Weissbach L, Harbrecht J, Kleinschmidt K, Schiefer HG, Friedrich HJ. Initial therapy of acute unilateral epididymitis using ofloxacin. I. Clinical and microbiological findings. Der Urologe. Ausg. A. 1990;29(5):272.

13. Owolabi AT, Fasubaa OB, Ogunniyi SO. Semen quality of male partners of infertile couples in Ile-Ife, Nigeria. Niger J Clin Pract. 2013;16:37-40. 
14. Akinola OI, Fabamwo AO, Rabiu KA, Akinoso OA. Semen quality in male partners of infertile couples in Lagos Nigeria. Int J Trop Med. 2010;5:37-9.

15. Ugboma HA, Obuna JA, Ugboma EW. Pattern of seminal fluid analysis among infertile couples in a secondary health facility in South-Eastern Nigeria. Res Obstet Gynecol. 2012;1:15-8.
16. Xie D, Klopukh B, Nehrenz GM, Gheiler E. Aspermia: A Review of Etiology and Treatment. Int Arch Urol Complic. 2017;3:023;1-8.

Cite this article as: Makwe CC, Ugwu AO, Ojewola RW, Onyeze CI. Seminal fluid parameters of male partners of infertile couples seeking care at the Lagos University teaching hospital. Int J Reprod Contracept Obstet Gynecol 2021;10:1347-51. 